\title{
Uso de pino de fibra de vidro para reabilitar unidade dental comprometida por extensa lesão cariosa: relato de caso
}

\author{
Use of fiberglass pin to rehabilitate dental unit compromised by extensive carious lesion: \\ case report
}

Uso de clavija de fibra de vidrio para rehabilitar unidad dental comprometida por lesión cariosa extensa: reporte de caso

Aurélio de Oliveira Rocha ${ }^{1 *}$, Thaine Oliveira Lima1, Lucas Menezes dos Anjos², Lucas Ferreira de Sá Santos ${ }^{1}$, Rafaela de Menezes dos Anjos Santos ${ }^{2}$, William José e Silva Filho², Cariorrana Vital de Sousa Macedoㄹ, Marcos Antônio Lima dos Santos², Rayle Monteiro Andrade², Nailson Silva Meneses Júnior².

\section{RESUMO}

Objetivo: Relatar um caso clínico onde a evolução da cárie causou comprometimento pulpar e estrutural em uma unidade dental que após tratamento endodôntico foi reabilitada com utilização de pino de fibra de vidro e restauração com resina composta. Detalhamento do caso: Paciente encaminhado para reabilitação oral pelo endodontista após realização previa de tratamento endodôntico da unidade dental 25 a qual se encontrava com extensa destruição coronal. Ao exame clínico inicial foi observado comprometimento considerável das paredes: oclusal, mesial e vestibular. Levando em consideração a queixa do paciente, análise oral clínica e limitações financeiras, foram propostas as seguintes etapas para tratamento: instalação de pino intrarradicular e posterior confecção de restauração direta em resina composta. O conduto foi parcialmente desobturado com posterior preparo das superfícies, cimentação do pino e restauração da unidade com resina composta devolvendo estética e função a paciente. Considerações finais: Os pinos de fibra de vidro têm demonstrado ótimo desempenho reabilitador, cabe ao dentista o conhecimento e aplicação de correta instalação desse componente intrarradicular.

Palavras-chave: Retentor intrarradicular, Odontologia, Restauração dentária.

\begin{abstract}
Objective: To report a clinical case where the evolution of caries caused pulp and structural impairment in a dental unit that, after endodontic treatment, was rehabilitated using a fiberglass pin and restoration with composite resin. Case details: Patient referred for oral rehabilitation by the endodontist after previous endodontic treatment of dental unit 25, which had extensive coronal destruction. Upon initial clinical examination, considerable involvement of the walls was observed: occlusal, mesial and vestibular. Taking into account the patient's complaint, clinical oral analysis and financial limitations, the following steps for treatment were proposed: installation of intraradicular pin and subsequent making of direct restoration in composite resin. The conduit was partially unclogged with subsequent preparation of the surfaces, cementation of the pin and restoration of the unit with composite resin, restoring aesthetics and function to the patient. Final considerations: The fiberglass pins have demonstrated excellent rehabilitation performance, it is up to the dentist to know and apply the correct installation of this intraradicular component.
\end{abstract}

Keywords: Intraradicular retainer, Dentistry, Dental restoration.

1 Universidade Tiradentes (UNIT), Aracaju - SE. *E-mail: aureliorocha2015@gmail.com

2 Universidade Federal de Sergipe (UFS), Aracaju - SE. 


\section{RESUMEN}

Objetivo: Presentar un caso clínico en el que la evolución de la caries provocó deterioro pulpar y estructural en una unidad odontológica que, luego de un tratamiento endodóntico, fue rehabilitada mediante un pin de fibra de vidrio y una restauración de resina compuesta. Detallando lo caso: Paciente remitido para rehabilitación oral por el endodoncista tras tratamiento endodóntico previo de la unidad dental 25, que presentaba una extensa destrucción coronal. En el examen clínico inicial se observó una afectación considerable de las paredes: oclusal, mesial y vestibular. Teniendo en cuenta la queja del paciente, el análisis clínico oral y las limitaciones económicas, se propusieron los siguientes pasos para el tratamiento: instalación de pin intrarradicular y posterior fabricación de restauración directa en resina compuesta. Se destapó parcialmente el conducto con posterior preparación de las superficies, cementación del perno y restauración de la unidad con resina compuesta, devolviendo la estética y función al paciente. Consideraciones finales: Los pines de fibra de vidrio han demostrado un excelente desempeño rehabilitador, corresponde al odontólogo conocer y aplicar la correcta instalación de este componente intrarradicular.

Palabras clave: Retenedor intradicular, Odontología, Restauración dental.

\section{INTRODUÇÃO}

A cárie dental é uma doença complexa e multifatorial associada a dieta cariogênica, má higiene dental, microorganismos e o tempo de interação entre esses fatores, a produção de ácidos por essas bactérias age diretamente nos tecidos minerais dos dentes culminando na dissolução e perda da estrutura. Essa patologia afeta indivíduos de todas as faixa-etárias, culturas, etnias e classes socioeconômicas sendo considerados fatores moduladores dessa condição (JARDIM JJ, et al., 2015; FEJERSKOV O, et al., 2015; SILVA BP, et al., 2018).

Inicialmente, a lesão de cárie apresenta-se como uma mancha branca localizada no esmalte, podendo evoluir para uma cavitação quando a dentina é afetada, o que causa comprometimento da estrutura dental. Quando sua progressão não é paralisada atingirá o complexo pulpar, necessitando assim de um tratamento endodôntico (RIBEIRO AA, 2016).

O tratamento endodôntico deve ser realizado como medida para eliminar e inativar os microrganismos e seus subprodutos permitindo reparação dos tecidos apicais, esse tratamento consiste no preparo químicomecânico do sistema de canais radiculares e vedação do mesmo por meio de materiais inertes (MARINIC K, et al., 2015).

Com a modernização no campo endodôntico, a limpeza e obturação dos canais radiculares de dentes com comprometimento pulpar causado por lesões cariosas profundas tem apresentado prognóstico cada vez mais promissores, contribuindo para a manutenção dessas unidades (BARBOSA BCTB, et al., 2016). Assim, há necessidade de utilizar uma técnica restauradora que seja igualmente benéfica para restabelecer a anatomia e estrutura perdida, favorecendo tanto a função quanto a estética desses elementos dentais. Assim, a busca por soluções satisfatórias em relação a retenção de materiais restauradores em dentes com grande perda do substrato dental e um aumento da realização de tratamento endodônticos (CLAVIJO V e KABBACH W, 2014).

Dentre as indicações dos pinos intrarradiculares podemos destacar: dentes com tratamento endodôntico e extensa perda tecidual, necessitando assim dessa ancoragem intrarradicular para retenção da restauração. Em relação ao modo de confecção esses pinos podem ser classificados em anatômicos ou pré-fabricados (SALES FGM, et al., 2018).

O pino anatômico ou também conhecido como núcleo metálico fundido fornece uma melhor adaptação aos canais excessivamente desgastados, são rígidos, e na maioria das vezes requer remoção mínima da estrutura dentária. Além disso, adaptam-se aos canais cônicos e com formato irregular, pois ele copia a anatomia individual do conduto radicular. Porém, o pino é fundido numa liga com módulo de elasticidade que pode ser dez vezes maior que o da dentina, e esta incompatibilidade com a transmissão de forças oclusais 
pode levar a fratura radicular. Além disso, esses pinos necessitam de etapas laboratoriais o que aumenta o tempo clínico da reabilitação oral e por terem coloração metálica podem comprometer a estética (TERRY DA e GELLER W, 2014).

Os pinos pré-fabricados ou pinos de fibra de vidro são considerados uma alternativa clínica aos dentes despolpados quando acometidos por extensa perda da estrutura dental. Por serem flexíveis e reforçados por fibras, esses pinos fraturariam antes do tecido dentário frente a uma extensa força oclusal, o que permite que o tratamento seja refeito sem comprometimento ou perda do dente. Além disso, reduz o número de consultas do paciente ao dentista, com excelentes resultados (PEREIRA JR, 2011).

Ademais, os pinos de fibra de vidro garantem função e geram uma harmonia estética ideal por apresentarem cor clara e transparência similar à dentina. Com isso eles proporcionam uma vida longa as restaurações e uma aparência mais similar ao dente natural (CLAVIJO V e KABBACH W, 2014).

Uma intervenção reabilitadora da estética e função dental necessita de um correto planejamento e interação entre as diversas especialidades odontológicas. A atualização do conhecimento, bem como o aperfeiçoamento das técnicas restauradoras, é fundamental na tomada de decisão para cada caso (DE OLIVEIRA ROCHA A, et al., 2021).

Assim, o objetivo desse estudo é relatar um caso clínico onde a evolução da cárie dental causou comprometimento endodôntico e estrutural em uma unidade dental que após tratamento endodôntico, foi reabilitada com utilização de pino de fibra de vidro e posterior restauração com resina composta.

\section{DETALHAMENTO DO CASO}

Paciente do sexo feminino, 23 anos, feoderma, procurou atendimento no consultório odontológico queixando-se da unidade dental 25 (segundo pré-molar esquerdo) que havia sido realizado tratamento endodôntico prévio com destruição extensa, sendo encaminhado pelo endodontista para reabilitação restauradora. Para realização do referente estudo a paciente assinou o Termo de Consentimento Livre e Esclarecido (TCLE) tendo assim conhecimento e autorizando o uso de suas imagens para fins acadêmicos. No aspecto inicial foi observado comprometimento considerável das paredes oclusal, mesial e vestibular (Figura 1).

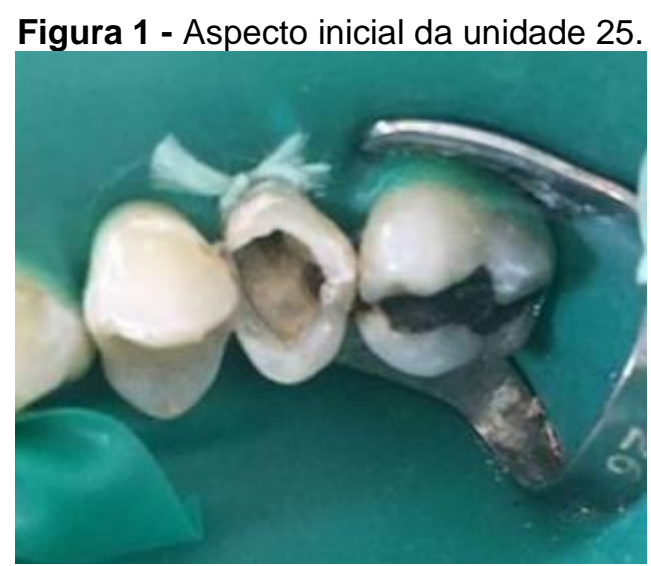

Fonte: Rocha AO, et al., 2021.

Levando em consideração a queixa do paciente, análise oral clínica e limitações financeiras, foram propostas as seguintes etapas para reabilitação: instalação de pino intrarradicular e posterior confecção de restauração direta em resina composta.

Inicialmente foi realizado radiografia periapical para avaliar 0 tratamento endodôntico e medir 0 comprimento do remanescente dental, o qual apresentou-se satisfatório e o comprimento radicular registrado foi de 13 milímetros (Figura 2). Seguindo o planejamento preconizado foi proposta a remoção $8 \mathrm{~mm}$ de gutapercha e permanência de $5 \mathrm{~mm}$ respeitando a quantidade mínima de $4 \mathrm{~mm}$. 
Antes do isolamento absoluto foi realizada profilaxia da unidade com pedra pomes (SS WHITE®) e água, seleção de cor de acordo com a escala vita classical (VITA®) onde foram registradas cores DA2 e EA2.

Foi realizado isolamento absoluto e remoção do curativo endodôntico para iniciarmos a desobturação parcial do conduto. Para a desobturação foram utilizadas brocas largo na seguinte sequência: broca 1, broca 2 e broca 3 (KG SORENSEN®) sempre com movimentos intermitentes e cuidadosos para não remover 0 material por completo além de remover todo o material das paredes circundantes.

Figura 2 - Radiografia inicial evidenciando tratamento endodôntico prévio satisfatório.

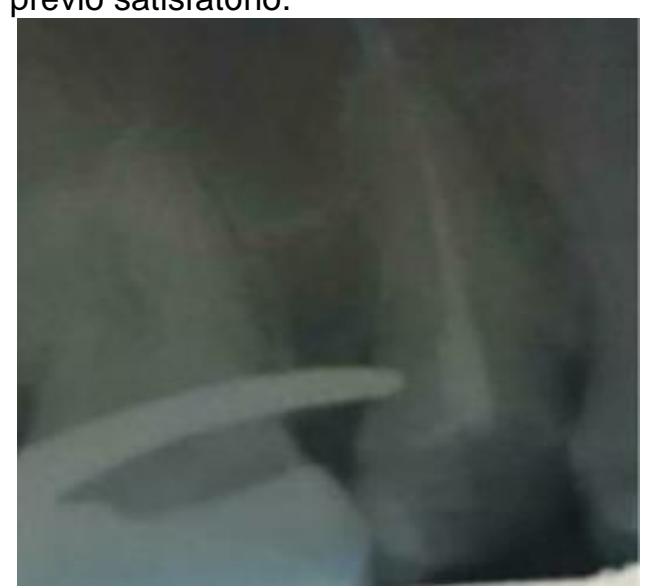

Fonte: Rocha AO, et al., 2021.

Após desobturação do conduto foi feita a seleção do pino por meio do teste de adaptação, sendo escolhido o pino número 1 (ANGELUS ${ }^{\circledR}$ ). Em seguida foi realizado o preparo do pino com ácido fosfórico a $37 \%$ (FGM®) durante 20 segundos para limpeza da superfície e abertura de poros superficiais. Em seguida foi aplicado silano (MAQUIRA®) por 60 segundos (Figura 3) para preparar a superfície que em seguida recebeu o adesivo (FGM®) que foi em seguida fotopolimerizado por 40 segundos (SDIß).

Figura 3 - Aplicação do silano na superfície do pino.

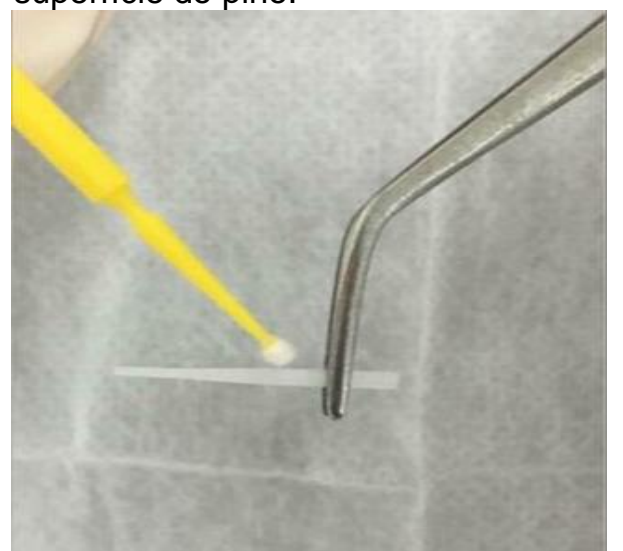

Fonte: Rocha AO, et al., 2021.

Após o preparo do pino foi realizado o preparo do conduto, para limpeza interna e desmineralização foi aplicado o ácido fosfórico $37 \%$ (FGM®) durante 20 segundos, em seguida o conduto foi lavado abundantemente por 40 segundos e seco com cones de papel absorventes. Uma camada de adesivo (FGM®) foi aplicada no interior do conduto. Foi introduzido no interior do conduto o cimento resiniso AllCem Core e em seguida foi encaixado o pino garantindo a posição correta para assim realizar a fotopolimerização (SDI®) do material. 
Após a foto ativação, o excesso do pino foi cortado com o uso de uma ponta esférica diamantada 1014 (KG SORENSEN®) em alta rotação e confeccionado o núcleo ao redor do mesmo utilizando o cimento resinoso.

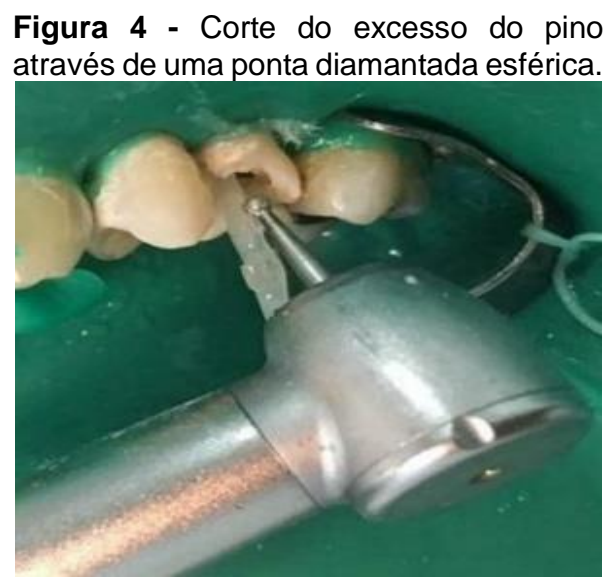

Fonte: Rocha AO, et al., 2021.

Após o corte do pino foi realizado o preparo o substrato dental remanescente da coroa com aplicação seletiva do ácido fosfórico $37 \%$ ( $F G M ®), 15$ segundos em dentina e 30 segundos em esmalte, lavagem abundante por 40 segundos, aplicação do adesivo $\left(F G M{ }^{\circledR}\right)$ e fotopolimerização (SDI®). Para restauração direta com resina composta $(F G M \circledast)$, uma matriz metálica foi utilizada para guiar na reconstrução das paredes mesial e vestibular. Inicialmente foi acomodado um incremento da resina EA2 a fim de devolver o ponto de contato mesial, em seguida foi colocado outro incremento igual para restabelecer a parede vestibular comprometida e em seguida foi utilizada a resina DA2 com acomodação de incrementos de até $2 \mathrm{~mm}$ de maneira oblíqua no interior da cavidade. A adição de resina composta por meio de incrementos visa reduzir contração de polimerização e falha da mesma. Foram fotopolimerizados (SDI®) cada incremento por vez.

Após a remoção do isolamento absoluto foi realizado ajuste oclusal para distribuição de contatos dentais seguido de acabamento da restauração com brocas multilaminadas (KG SORENSEN®), pontas abrasivas e tiras de lixa (AMERICA BURS $®$ ) evidenciando o aspecto final imediato da restauração (Figura 5A). Por fim foi realizada uma tomada radiográfica para evidenciar adaptação do pino e restauração que se apresentaram excelentes radiograficamente (Figura 5B).

Figura 5 - Restauração e aspecto final.

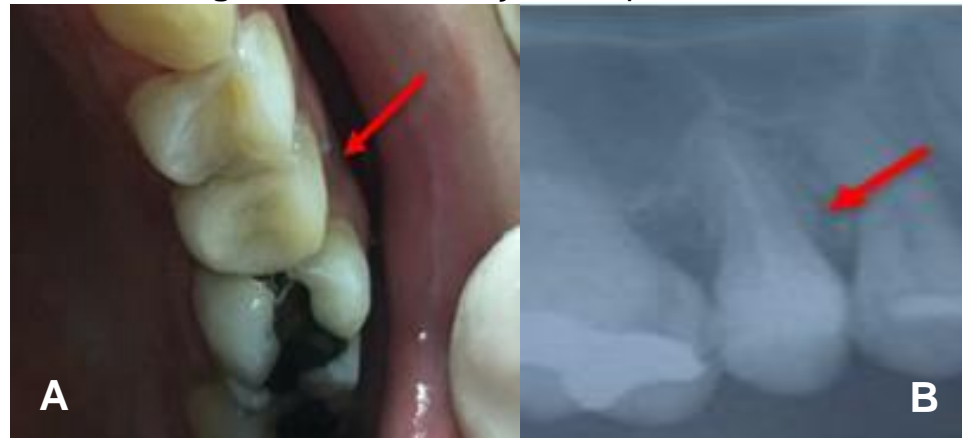

Legenda: A - Aspecto final imediato da restauração.

B - Aspecto radiográfico do pino e restauração.

Fonte: Rocha AO, et al., 2021.

A paciente retornou após sete dias para avaliação e polimento final, clinicamente a restauração estava satisfatória, sem queixa, sinal de fratura ou infiltração marginal.

\section{DISCUSSÃO}

A perda precoce de dentes é cada vez menos preferível pelos pacientes, além da importância funcional os dentes refletem a estética e a beleza. A busca por procedimentos reabilitadores de dentes acometidos por 
injúrias é uma rotina na prática clínica diária. Com o avanço, modernização e conhecimentos nos campos odontológicos surgem materiais e técnicas cada vez menos invasivas e que garantem a permanência dos dentes naturais na cavidade bucal (BARBOSA BCTB, et al., 2016). A paciente em questão desejava principalmente que o dente fosse reabilitado ao invés de extraido, não mencionando em momento algum a perda da unidade dental.

A reabilitação de dentes tratados endodonticamente com grande destruição coronária tem o objetivo de devolver forma, estética e função dos elementos dentais. Para que esses objetivos sejam alcançados, é necessária a utilização de materiais que possibilitem a retenção da restauração e que promovam proteção da estrutura dentária, prevenindo a ocorrência de fraturas e de cárie (FARINA AP, et al., 2011). O dente reabilitado exibia uma destruição coronária considerável apresentado perda das paredes: oclusal, mesial e vestibular.

O tratamento restaurador pode ser feito de forma direta, semi-direta ou indireta em dentes tratados endodonticamente, podendo necessitar ou não de algum tipo de retentor intraradicular para que aumente a retenção do material restaurador devido aumento da área de contato para adesão, principalmente em dentes que possuem pouco remanescente dental como no caso da perda de muitas paredes (BARATIERI LN, et al., 2010). Tendo em vista as formas de reabilitar um dente com perda extensa de substrato optou-se por lançar mão da técnica restauradora direta, pois esta, demanda menos custos e menor tempo de confecção, critérios comentados pela paciente como importantes em seu caso. Além disso, com a evolução dos materiais restauradores a resina composta tem se mostrado cada vez mais eficiente nos quesitos resistência e estética para uso de forma direta.

Até pouco tempo os núcleos metálicos fundidos eram a única opção para restauração de dentes endodonticamente tratados, contudo apresentam limitações na sua indicação, dentre as quais incluem a tensão gerada na estrutura dentária, devido ao seu alto módulo de elasticidade cerca de dez vezes maior que o da dentina natural, podem ocasionar fraturas radiculares e o fator estético, onde a luz incidente é completamente bloqueada pelo pino metálico (CLAVIJO V e KABBACH W, 2014; SALES FGM, et al., 2018; MIORANDO B, et al., 2018). Esses fatores negativos associados aos núcleos metálicos fundidos foram determinantes para não utilizar esse componente no caso clínico exposto, além da necessidade de uma etapa laboratorial que demandaria maior tempo e maior investimento financeiro da paciente.

Com a finalidade de aumentar a área de contato das restaurações diretas com o substrato dental os pinos de fibra de vidro têm sido amplamente utilizados (LAMICHHANE A, et al., 2014). Esses garantem uma maior distribuição das tensões durante movimentos funcionais como a mastigação, já que seu módulo de elasticidade é similar ao do tecido dentinário, diminuindo o risco de fraturas radiculares (BARBOSA BCTB, et al., 2016). A utilização do pino no referente caso clínico teve como principal intuito as características citadas, ou seja, aumentar a área de superfície do substrato para adesão da resina, além de proteger o remanescente em caso de grande força onde o pino fraturaria antes do dente.

Interligada a essas características, ainda apresentam a vantagem de boa estética quando comparado aos pinos de coloração metálica e não necessitam de trabalho laboratorial para confecção, diminuindo o tempo clínico além de permitir um preparo mais conservador da estrutura dental (BARBOSA BCTB, et al., 2016; VON STEIN-LAUSNITZ M, et al., 2019). De acordo com as vantagens discutidas sobre o pino de fibra de vidro esse foi o material de escolha para reabilitar a unidade 25 , os pré-molares quando expostos ao sorriso são considerados em área estética, como no caso da paciente tratada.

A desobturação do conduto radicular pode ser realizada por meio da técnica manual ou mecanizada. A técnica manual consiste na remoção do material obturador através do uso de instrumentos manuais como limas $\mathrm{K}$ em combinação com solventes, porém os solventes podem ser citotóxicos e trazer algumas complicações se entrar em contato com o paciente. A técnica mecanizada consiste na remoção do material por meio do uso de brocas como a largo em baixa rotação, porém essa técnica deve ser feita com cautela para não remover todo o material (MAUTONE EP, et al., 2014). Tendo em vista as limitações e complicações associadas a técnica manual, a técnica mecanizada foi escolhida para desobturação no presente caso clínico. O preparo prévio do substrato para cimentação deve ser realizado por meio do condicionamento com ácido 
fosfórico 37\% para limpeza da superfície pois o ácido remove a lama dentinária e criação de microporosidades superficiais que serão preechidas por adesivo garantindo a hibridização dentinária (SALES IVM, et al., 2021). Como preconizada pela literatura essa foi a técnica empregada para o tratamento do substrato no caso relatado.

O cimento Allcem Core possui dupla cura, ou seja, seu endurecimento ocorre pela ação da luz (cura química) e pela ação do tempo (cura física) o que garante que onde a luz do fotopolimerizador não chegar de forma ativa seja endurecido pela ação do tempo (MAGALHÃES FILHO TR, 2019). Levando em consideração suas ótimas características esse foi o material de escolha para cimentação do caso discutido.

A Odontologia reabilitadora não é atribuição restrita de especialistas, também está presente na prática diária e vivência do clínico geral. O conhecimento e respeito das técnicas e propriedades dos materiais dentários são o segredo para um excelente desempenho e altas taxas de sucesso clínico. Os procedimentos reabilitadores estéticos e funcionais requerem observação, conhecimento e aplicação cuidadosa das técnicas e protocolos preconizados (NAMORATTO LR, et al., 2013; GUERRA M, et al., 2017). Os pinos de fibra de vidro têm demonstrado ótimo desempenho reabilitador, cabe ao dentista o conhecimento e aplicação de correta instalação desse componente intrarradicular.

A reabilitação da unidade dental do presente caso clínico devolveu para a paciente sua estética e função, ou seja, proporcionou qualidade de vida, o pino de fibra de vidro quando utilizado de forma correta garante estética e estrutura suficiente para adesão do material restaurador, que por sua vez deve ser acomodado e esculpido respeitando a anatomia dental e o tecido gengival o que culminará em uma maior taxa de sucesso do procedimento e conforto para o paciente.

\section{REFERÊNCIAS}

1. BARATIERI LN, et al. Odontologia restauradora: fundamentos e técnicas. São Pulo: Editora Santos, 2010.

2. BARBOSA BCTB, et al. Pinos de fibra: revisão de literatura. Uningá Review, 2016; 28(1): 83-7.

3. CLAVIJO V, KABBACH W. Pinos anatômicos: acredite nessa técnica. Clinica-International Journal of Brazilian Dentistry, 2014; 10(1): 12-21.

4. DE OLIVEIRA ROCHA A, et al. Intervenção estética anterior por meio de facetas diretas em resina composta: relato de caso. Revista Eletrônica Acervo Saúde, 2021; 13(3).

5. FARINA AP, et al. Bond strength of fiber posts in different root thirds using resin cement. J Adhes Dent, 2011; 13(2): 179-86.

6. FEJERSKOV O, et al. Dental Caries: The Disease and Its Clinical Management. 3rd Ed. Oxford (UK): Wiley Blackwell, 2015.

7. GUERRA MLRS, et al. Fechamento de diastemas anteriores com resina composta direta: relato de caso. Faculdade de Odontologia de Lins/Unimep, 2017; 27(1): 63-68.

8. JARDIM JJ, et al. Remoção parcial de tecido cariado em dentes permanentes: seis anos de acompanhamento. Revista da Faculdade de Odontologia-UPF, 2015; 20(1).

9. LAMICHHANE A, et al. Dental fiber-post resin base material: a review. The Journal of advanced prosthodontics, $2014 ; 6(1): 60$.

10. MAGALHÃES FILHO TR. Análise comparativa das propriedades mecânicas de cimentos resinosos convencionais e autoadesivos. Revista Científica da UNIFENAS, 2019; 1(2).

11. MAUTONE EP, et al. Desobturação e repreparo do canal radicular: análise da eficácia de duas técnicas preconizadas. Rev Odontol Bras Central, 2014; 23(64), 58-64.

12. MARINIC K, et al. Repeated exposures to blue light--activated eosin $Y$ enhance inactivation of $E$. faecalis biofilms, in vitro. Photodiagnosis and Photodynamic Therapy, 2015; 12: 393-400.

13. MIORANDO B, et al. Utilização de pinos intrarradiculares. J Res Dent, 2018; 6(1): 16-22

14. NAMORATTO LR, et al. Cementation in ceramics: improvement of conventional and adhesive procedures. Revista Brasileira de Odontologia, 2013; 70(2): 142-147.

15. PEREIRA JR. Retentores Intrarradiculares. Artes Médicas, 2011.

16. RIBEIRO AA. Biological Features of Dental Caries. JSM Dent, 2016; (3): 1065-70.

17. SALES FGM, et al. Reconstrução coronária com pino de fibra de vidro: relato de caso. Archives of Heal Inv, $2018 ; 7$.

18. SALES IVM, et al. Tratamento endodôntico com instalação de pino de fibra de vidro anatomizado: relato de caso. Brazilian Journal of Development, 2021; 7(5).

19. TERRY DA, GELLER W. Odontologia estética e restauradora. 2 ed. São Paulo: Editora Quintessence, 2014.

20. VON STEIN-LAUSNITZ M. Direct restoration of endodontically treated maxillary central incisors: post or no post at all?. Clinical oral investigations, 2019; 23(1): 381-389. 